\title{
Identifying critical state of complex diseases by single-sample Kullback-Leibler divergence
}

\author{
Jiayuan Zhong, Rui Liu* and Pei Chen ${ }^{*}$
}

\begin{abstract}
Background: Developing effective strategies for signaling the pre-disease state of complex diseases, a state with high susceptibility before the disease onset or deterioration, is urgently needed because such state usually followed by a catastrophic transition into a worse stage of disease. However, it is a challenging task to identify such predisease state or tipping point in clinics, where only one single sample is available and thus results in the failure of most statistic approaches.

Methods: In this study, we presented a single-sample-based computational method to detect the early-warning signal of critical transition during the progression of complex diseases. Specifically, given a set of reference samples which were regarded as background, a novel index called single-sample Kullback-Leibler divergence (sKLD), was proposed to explore and quantify the disturbance on the background caused by a case sample. The pre-disease state is then signaled by the significant change of sKLD.

Results: The novel algorithm was developed and applied to both numerical simulation and real datasets, including lung squamous cell carcinoma, lung adenocarcinoma, stomach adenocarcinoma, thyroid carcinoma, colon adenocarcinoma, and acute lung injury. The successful identification of pre-disease states and the corresponding dynamical network biomarkers for all six datasets validated the effectiveness and accuracy of our method.

Conclusions: The proposed method effectively explores and quantifies the disturbance on the background caused by a case sample, and thus characterizes the criticality of a biological system. Our method not only identifies the critical state or tipping point at a single sample level, but also provides the SKLD-signaling markers for further practical application. It is therefore of great potential in personalized pre-disease diagnosis.
\end{abstract}

Keywords: Tipping point, Dynamic network biomarker (DNB), Pre-disease state, Critical transition, Single-sample Kullback-Leibler divergence (sKLD)

\section{Background}

Critical transitions are sudden and large-scale state transitions that occur in many complex systems, such as ecological systems [1,2], climate systems [3, 4], financial markets [5, 6], microorganism populations [7], psychiatric conditions [8],infectious disease spreading [9] and the human body [10]. Recently, considerable evidence suggests that during the progression of many complex diseases, e.g. cancer [11], asthma attacks [12], epileptic seizures [13] the deterioration is not always smooth but

\footnotetext{
* Correspondence: scliurui@scut.edu.cn; chenpei@scut.edu.cn

School of Mathematics, South China University of Technology, Guangzhou 510640, China
}

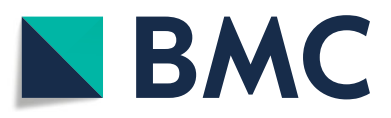

(c) The Author(s). 2020 Open Access This article is distributed under the terms of the Creative Commons Attribution 4.0 International License (http://creativecommons.org/licenses/by/4.0/), which permits unrestricted use, distribution, and

reproduction in any medium, provided you give appropriate credit to the original author(s) and the source, provide a link to the Creative Commons license, and indicate if changes were made. The Creative Commons Public Domain Dedication waiver (http://creativecommons.org/publicdomain/zero/1.0/) applies to the data made available in this article, unless otherwise stated. point, at which a drastic or quatitative tansition may point, at which a drastic or qualitative transition may occur. Accordingly, the progression of a complex disease can be roughly divided into three stages regardless of specific biological and pathological differences during the progression of diseases, that is, (1) a normal state, a steady state representing the relatively healthy stage and with high resilience; (2) a pre-disease state, which is the limit of the normal state immediately before the onset of deterioration, and with low resilience and high susceptibility; and (3) a disease state, the other steady state with high resilience after the qualitative deterioration (Fig. 1a). It is important to predict the tipping point, so as to 


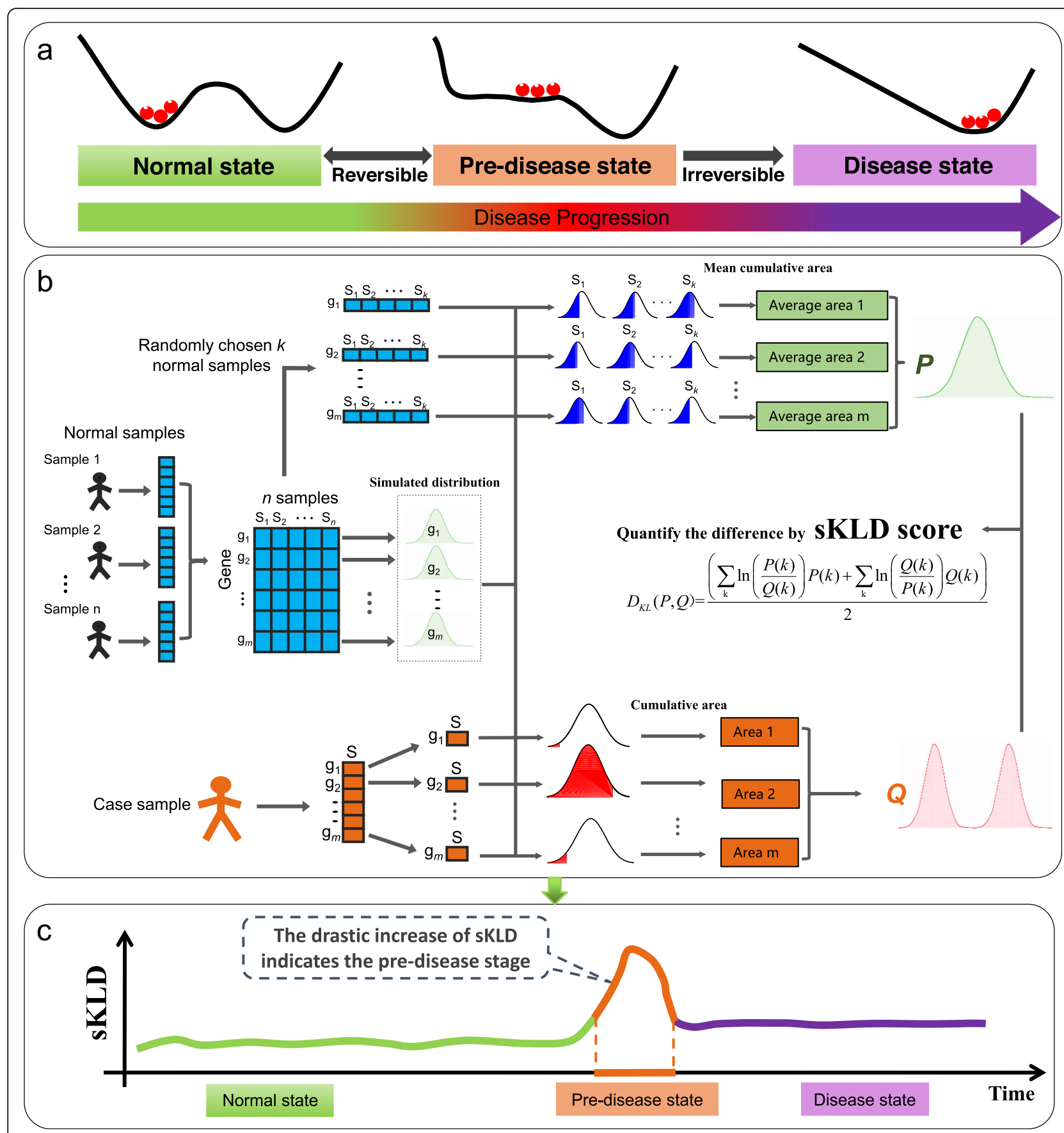

Fig. 1 The outline for detecting the early-warning signal of a pre-disease state based on SKLD. a The progression of complex diseases is modeled as three states, including two stable states, i.e., a normal and a disease state with high stability and resilience, and an unstable pre-disease state with low stability and resilience $[5,9]$. As the limit of the normal state, the pre-disease state is a critical state just before the onset of deterioration. b Given a number of reference samples that are generally from normal cohort and represent the healthy or relatively healthy individuals, the sKLD score is capable to quantitatively evaluate the difference between two distributions of each gene, i.e., the background distribution that generated from a set of reference samples, and a perturbed distribution yielded from the single case sample. The detailed procedure and description of deriving the two distributions are presented in Methods section. c During the progression of a complex disease, the pre-disease state is indicated by the significant change of sKLD, i.e., the sKLD changes gradually when the system is in the normal state, while it increases abruptly when the system approaches the tipping point 
prevent or at least get ready for the upcoming deterioration by taking appropriate intervention actions. Recently, we proposed a theoretic framework, called the dynamical network biomarker (DNB) concept [10, 14] for identifying the pre-disease state of complex diseases. This DNB concept, directly from the critical slowingdown theory $[15,16]$, provides statistical method to select relevant variables for the pre-disease state, that is, a small group of closely related variables (DNBs) convey early warning signals for the impending critical transition by some drastic statistical indices [17, 18]. The DNB theory and its extensions have been applied to several cases, detected the tipping points of endocrine resistance [19] as well as cellular differentiation [20], investigated the immune checkpoint blockade [21], and helped to find the corresponding pre-disease states of several diseases $[18,22-26]$. However, DNB method requires multiple samples at each time point, which are generally not available in clinics and other practical cases, thus significantly restricting the application of DNB method in most real cases. Therefore, when there is only a single case sample available, it requires new computational method to explore the critical information, detect the early-warning signal and identify the pre-disease state.

The rapid development of high-throughput technology provides new insights for computational analysis, even when there is only one single sample available. Actually, based on a sample of high-throughput data, it is possible to measure the expressions of thousands of genes simultaneously. Such high-dimensional observation at the genome-wide scale not only provides the global view of a biological system, but also presents the accumulated effects of its long-term dynamics. Motivated by this point, in this study we develop a data-driven computational method and achieve the single-sample detection of the pre-disease state, by exploring the rich dynamical information from the high-throughput omics data. Specifically, it is found that the qualitative state change often causes the significant changes in the distributions of some genes' expression. Therefore, a novel index, the single-sample Kullback-Leibler divergence (sKLD), is proposed to quantify the disturbance brought by the single case sample on the background distribution, where the background or reference samples refer to samples collected from a few healthy/relatively healthy individuals. Correspondingly, an applicable algorithm is developed based on sKLD (Fig. 1b), including a procedure of simulating the background distribution for each gene, evaluating the perturbation to the background distribution triggered by a single case sample, detecting the early-warning signal and identifying the pre-disease state. During this procedure, a group of biomolecules whose expressions are highly fluctuating before the critical transition are also picked out as the sKLDsignaling marker for further practical application. This new approach has been applied to a numerical simulation, and six real datasets including lung squamous cell carcinoma (LUSC), lung adenocarcinoma (LUAD), stomach adenocarcinoma (STAD), thyroid carcinoma (THCA), colon adenocarcinoma (COAD) from the cancer genome atlas (TCGA) database and acute lung injury (GSE2565) from the NCBI GEO database. The identified pre-disease states all agree with the experimental observation or survival analysis. And the corresponding signaling markers have been validated by functional enrichment.

\section{Results}

We present the definition and algorithm of sKLD score in Methods section. Here, we used a single-sample with high-throughput omics data, to identify the pre-disease state or early warning signals of the disease deterioration based on the sKLD score. Achieving reliable identification with only one sample is of great importance in clinic application since it is usually difficult to obtain multiple samples from an individual who does not yet exhibit any disease symptoms during a short period. To illustrate how sKLD works, we applied our method first to a simulated dataset, and then to six real datasets, including LUSC, LUAD, STAD, THCA and COAD from TCGA database (http://cancergenome.nih.gov) and acute lung injury (GSE2565) from the GEO database (http:// www.ncbi.nlm.nih.gov/geo/). The successful identification of the pre-disease states in these diseases validated the effectiveness of sKLD method in quantifying the tipping point just before the critical transitions into severe disease states.

\section{Validation based on numerical simulation}

A model of an eight-node artificial network (Fig. 2a) was used to validate the proposed computational method. This network is the regulatory representation for a set of eight biomolecules, governed by eight stochastic differential equations Eq. (S1) shown in Additional file 1: A. Such a model is represented in Michaelis-Menten form. This type of regulatory network is usually applied to study genetic regulations including transcription and translation processes [27-29], and multi-stability and nonlinear biological processes [30, 31]. In addition, the bifurcation in Michaelis-Menten form is often employed to model the state transition of gene regulatory networks $[32,33]$. In Eq. (S1), a parameter $s$ was varying from 0.5 to 0.2 . Based on this model, a numerical simulation dataset was generated.

It is seen in Fig. $2 b$ that the single-sample Kullback-Leibler divergence (sKLD) abrupt increases when the system approaches a special parametric value $s=0$, which was set 

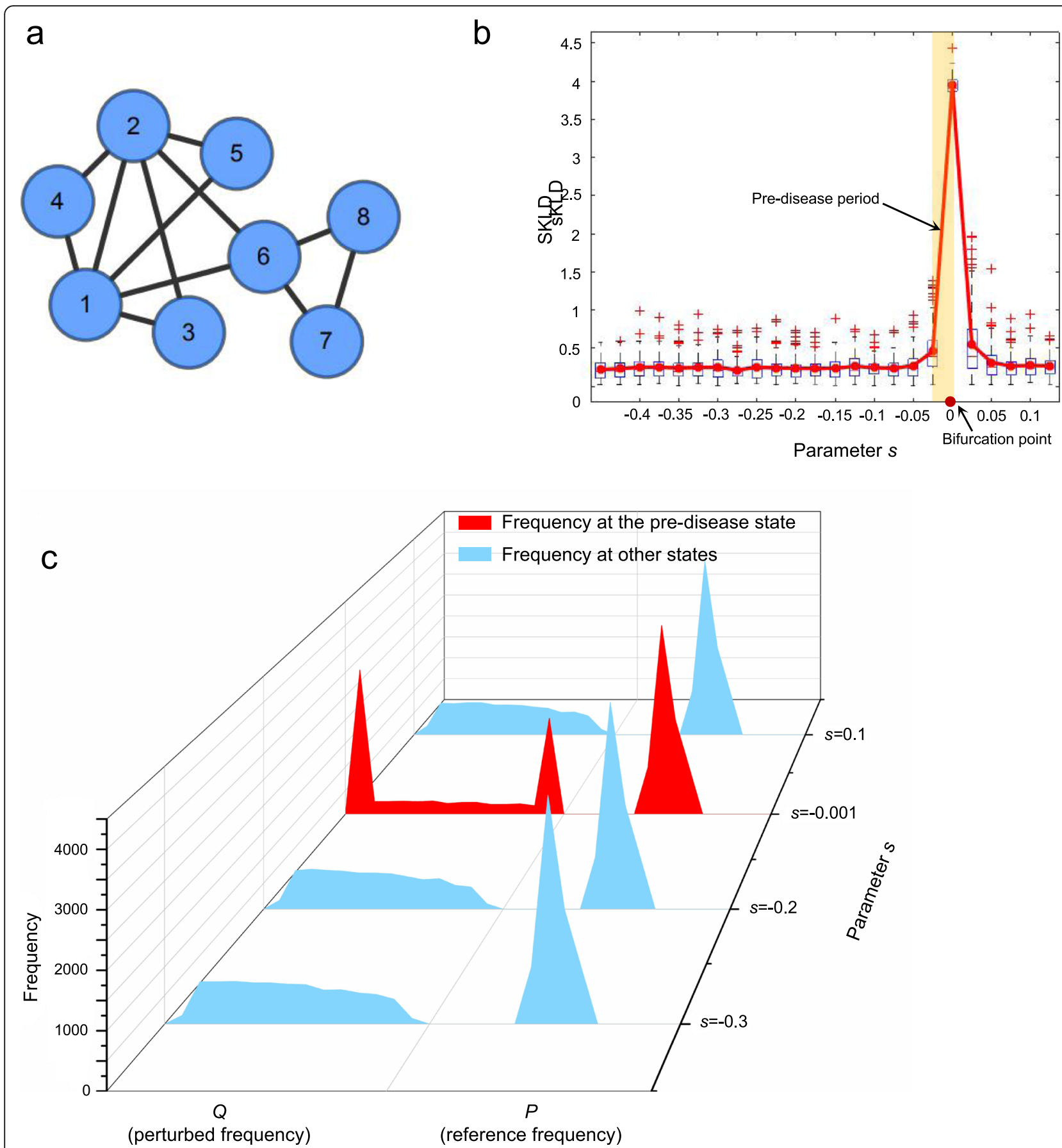

Fig. 2 The performance of sKLD based on a dataset of numerical simulation. a A network with eight nodes governed by a model is represented in Michaelis-Menten form, based on which the numerical simulation is conducted. $\mathbf{b}$ The curve of SKLD score defined in Eq. (2). It is obvious that the sKLD would abruptly increase when the system is near the critical point, i.e., $s=0$, which is in accordance with the bifurcation parameter value at $s=0$ (see Eq. (S3) in Additional file 1: A). $\mathbf{c}$ It is seen that the perturbed frequency Q presents two peaks when the system approaches the tipping point, i.e., $s=0$, comparing with that in a normal state $(s=-0.2)$ or a disease state $(s=0.1)$ and there is no significant difference in three stages of disease progression for the reference $P$

as a Hopf bifurcation value (see Additional file 1: A for details). In other word, the high level of sKLD in the vicinity of the critical parameter value $s=0$ represents that the reference distribution $P$ is significantly different from the perturbed distribution $Q$, which was generated from a single pre-disease sample. Besides, to demonstrate the robustness of the proposed method, a hundred SKLD scores were calculated for each parameter $s$, respectively based 
on a hundred single samples perturbed by additive white noise. It is seen that the median values of the box plots in Fig. $2 \mathrm{~b}$ also stably provides signals for the tipping point, which indicates the sKLD score is featured with robustness against sample noises. To better illustrate the different distribution between normal and pre-disease states, the dynamical progression of frequencies $P$ and $Q$ were demonstrated in Fig. 2c with a series of parametric values, i.e., $s \in\{-0.3,-0.2,-0.001,0.1\}$. Each frequency in Fig. $2 \mathrm{c}$ is a statistical plot based on ten thousand simulations. From these frequency plots, it suggests that the perturbed frequency $Q$ in a pre-disease state $(s=0)$ presents two peaks, that is, when the network system is in a pre-disease state, the expressions of some nodes wildly fluctuate in a strongly collective manner, resulting a distinct distribution. This critical phenomenon is accurately detected by sKLD, which quantitatively provides a score for identifying the upcoming bifurcation point. Therefore, the numerical simulation validated the effectiveness of SKLD in detecting the early warning signal of a qualitative state transition. The detailed dynamical system is proposed in Additional file 1: A. The source code of numerical simulation is accessed in https://github.com/zhongjiayuna/KL_Project.

\section{Identifying the critical transition for acute lung injury}

The sKLD has been applied to the microarray data of dataset GSE2565, which is obtained from a mouse experiment of phosgene-induced acute lung injury [34]. In the original experiment, the gene expression data of case samples were derived from the lung tissues of $\mathrm{CD}-1$ male mice exposed to phosgene up to $72 \mathrm{~h}$, while the data of control samples were from that exposed to air. During the experiment for both case and control groups, there are totally nine sampling points, i.e., $0,0.5,1,4,8$, $12,24,48$, and $72 \mathrm{~h}$, while at each sampling time point, lung tissues were obtained from six mice [34]. Applying the proposed sKLD-based method to the dataset, we regard the six samples at the first time point $(0 \mathrm{~h})$ as the reference/normal samples for both case and control groups. The mean sKLD score shown as the red curve in Fig. 3a, abruptly increases and reaches a peak at $8 \mathrm{~h}$, suggesting that there is a critical transition around $8 \mathrm{~h}$. To demonstrate the significance of the result, six datasets were generated from a leave-one-out scheme. Applying the sKLD algorithm to these datasets respectively, six mean sKLD scores were derived and plotted as the yellow curves in Fig. 3a. It is seen that these sKLD curves based on the re-sampled datasets all indicates the tipping point at $8 \mathrm{~h}$. In Fig. 3b, it exhibits the dynamical change of distributions for both case and control samples. Obviously for control samples, there is little dynamical difference in the perturbed distributions, while for case samples, the perturbed distribution at the 4th sampling time point $(8 \mathrm{~h})$ is notably distinct from that at other sampling time points (Fig. 3b), leading to the significant change of sKLD score of case samples at $8 \mathrm{~h}$. The abrupt change of such quantitative index demonstrates its effectiveness in detecting early signals of critical transition for complex diseases at a network level, which may also reveal the mechanisms on disease progression [35-37]. In Fig. 3c, we demonstrate the dynamical evolution of a network composed by the top $5 \%$ most significant genes in terms of the cumulative area of the case sample. Clearly, an obvious change in the network structure occurs around $8 \mathrm{~h}$, signaling the upcoming critical transition at the network level. These results agree with the observation in original experiment, that is, after 8-h exposure to phosgene, the mice in case group were observed a series of symptoms including enhanced bronchoalveolar lavage fluid (BALF) protein levels, increased pulmonary edema, and ultimately decreased survival rates [34]. The severe phosgene-induced acute lung injury is around $8 \mathrm{~h}$ and lasts until $12 \mathrm{~h}$ after exposure. About $50-60 \%$ deaths were observed after 12 hous exposure, and $60-70 \%$ mortality was observed after 24-h exposure [34]. Comparing with the former DNB method [10], the common signaling genes for acute lung injury is provided in Additional file 3.

\section{Identifying the critical transition for tumor diseases}

To demonstrate the effectiveness, the proposed sKLD method is applied to five tumor datasets, lung squamous cell carcinoma (LUSC), lung adenocarcinoma (LUAD), stomach adenocarcinoma (STAD), thyroid carcinoma (THCA), colon adenocarcinoma (COAD) from the cancer genome atlas (TCGA), all of which were composed by tumor and tumor-adjacent samples. The tumor samples were grouped into different cancer stages according to corresponding clinical information of TCGA, that is, the tumor samples were classified into seven stages for LUSC, LUAD and STAD, and four stages for THCA and COAD. The detailed sampling conditions are provided in Additional file 1: Table S1. In all the five datasets, the tumoradjacent samples were employed as normal/reference samples. The sKLD was then calculated for each single tumor sample following the proposed algorithm (the five steps) in Methods. Finally, the average sKLD of each stage was taken to identify any possible critical/pre-transition state.

Clearly, the significant change of sKLD successfully indicated the critical stages prior to the metastasis for all the five cancers (Fig. 4a-e). To validate the identified critical state, the prognosis results respectively based on before-transition and after-transition samples were exhibited and compared through Kaplan-Meier (log-rank) survival analysis (Fig. 4f-j and Additional file 1: Figure S4). Specifically, before the identified critical stage, there is generally a high expectation of life after diagnosis, while after the critical stage, there is a much lower 
a

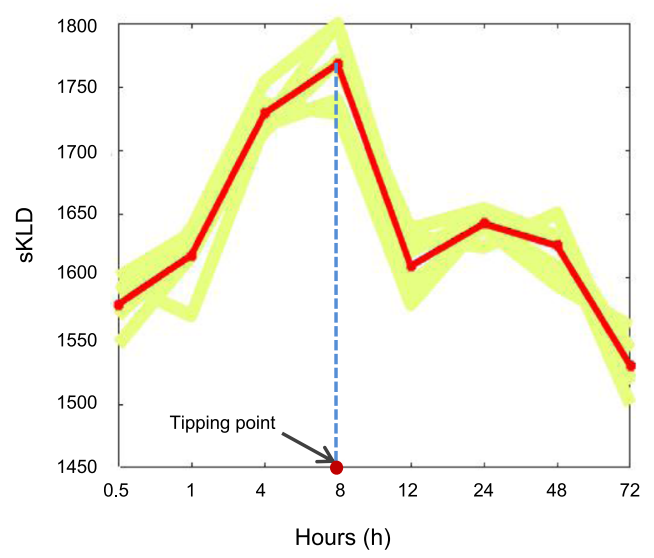

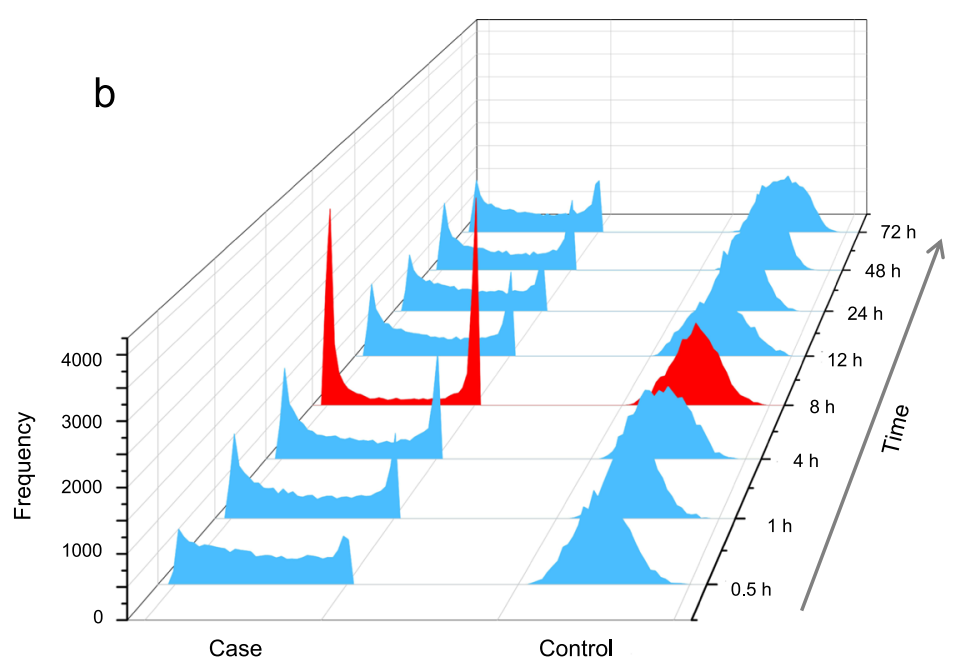

C
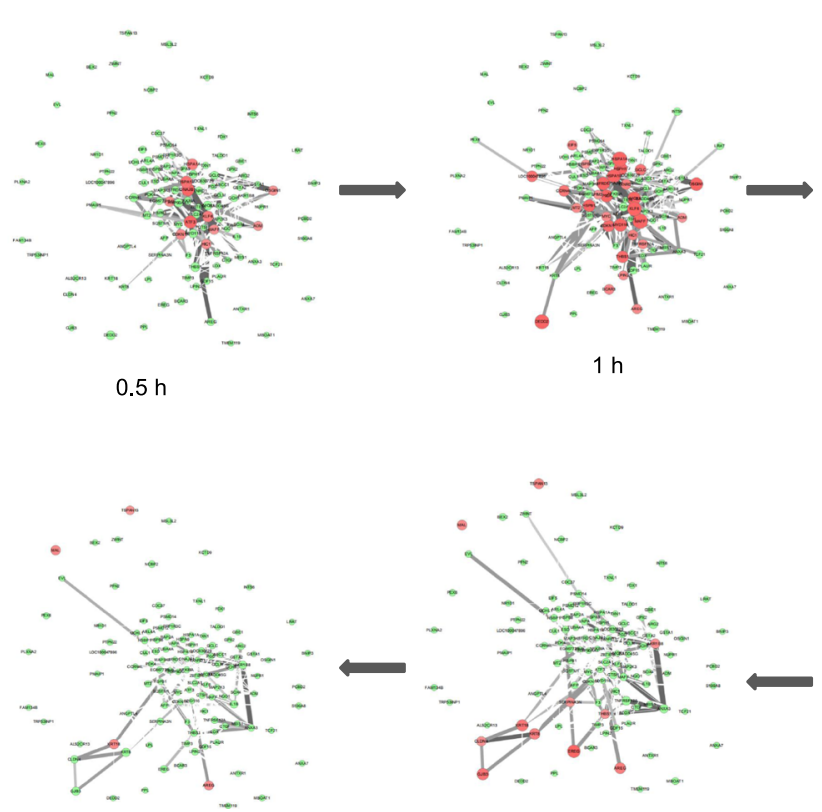

$72 \mathrm{~h}$
$1 \mathrm{~h}$

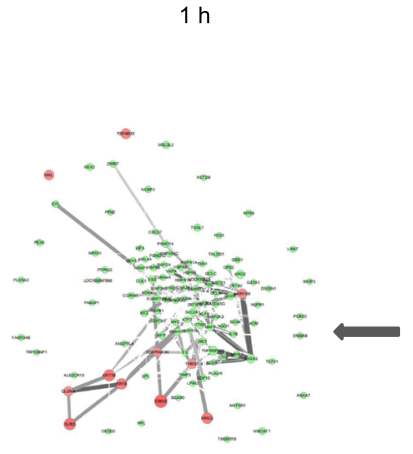

$48 \mathrm{~h}$

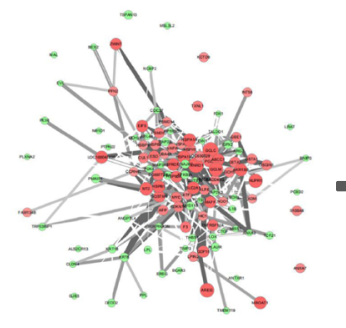

$4 \mathrm{~h}$

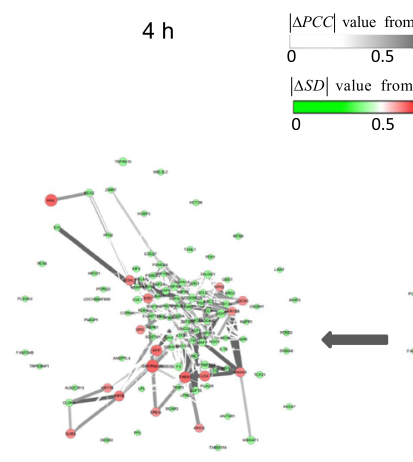

$24 \mathrm{~h}$

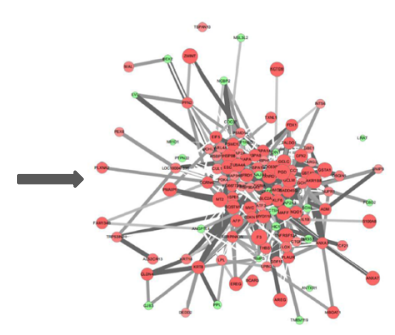

$\begin{array}{cc}\triangle P C C \mid \text { value from } 0 \text { to } 1 & 8 \mathrm{~h}\end{array}$

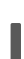

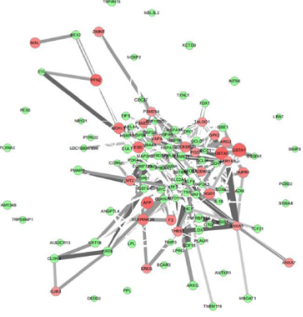

$12 \mathrm{~h}$

Fig. 3 The application of sKLD in acute lung injury. a As shown in the red curve, the peak for the sKLD appears at $8 \mathrm{~h}$, which can be used as an early signal of acute lung injury deterioration. The result is consistent with the experimental observation. To illustrate the significance of the result, six yellow curves are derived based on six sets of datasets generated from a leave-one-out scheme, which consistently indicate the tipping point at $8 \mathrm{~h}$. $\mathbf{b}$ The figure shows the dynamical changes in the distribution of signaling genes for the case data and control data, respectively. $\mathbf{c}$ From the dynamical evolution of the network composed by the top $5 \%$ most significant genes in terms of the cumulative area of the case sample, it is seen that the an obvious change in the network structure appear at $8 \mathrm{~h}$

expectation of survival after diagnosis (Fig. 4f-j). However, before and after any other stages, there was no significant difference in the prognosis (Additional file 1: Figure S4), which suggests that the identified critical stage is accurate and closely associated with prognosis.

\section{The critical state of LUSC}

For LUSC, the sKLD score abruptly increases at stage IIA (Fig. 4a), indicating an upcoming critical transition after stage IIA, that is, the invasion into the mediastinal pleura at stage IIB, after which there are lymph nodes metastasis, tumor invaded the visceral pericardial surface and the intrapericardial pulmonary artery [38]. The critical transition has also been validated by survival analysis. It is seen from Fig. 4f that the survival time of before-transition samples (samples from stages IA-IIA) is much longer than that of after-transition samples (samples from stages IIB-IV), resulting significant 

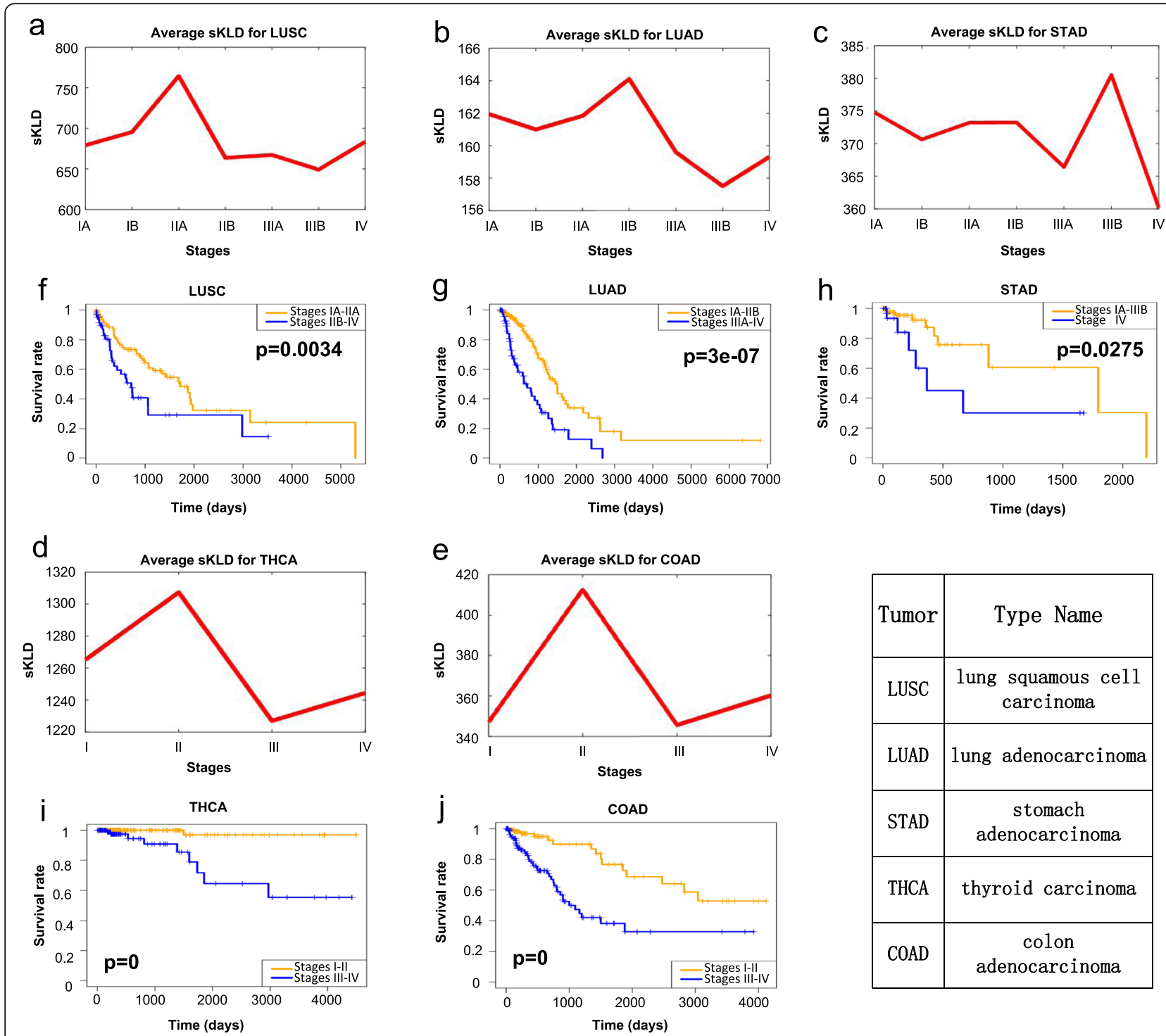

\begin{tabular}{|c|c|}
\hline Tumor & Type Name \\
\hline LUSC & $\begin{array}{c}\text { lung squamous cell } \\
\text { carcinoma }\end{array}$ \\
\hline LUAD & lung adenocarcinoma \\
\hline STAD & $\begin{array}{c}\text { stomach } \\
\text { adenocarcinoma }\end{array}$ \\
\hline THCA & $\begin{array}{c}\text { thyroid carcinoma } \\
\text { COAD }\end{array}$ \\
adenocarcinoma \\
\hline
\end{tabular}

Fig. 4 Identification of critical transition for tumor deterioration in five cancers: a LUSC, b LUAD, c STAD, $\mathbf{d}$ THCA and e COAD. Comparison of survival curves before and after critical state for five cancers: $\mathbf{f} L U S C, \mathbf{g} L U A D, \mathbf{h} S T A D, \mathbf{i} T H C A$ and $\mathbf{j} C O A D$

difference (significant value $p=0.0034$ ) between the survival curves of two sets of samples, i.e., samples derived before and after stage IIA of LUSC. For the samples solely from the two stages around the critical transition point, i.e., stages IIA and IIB, the survival time of stageIIA samples is longer than that of stage-IIB samples $(p=0.036$; Additional file 1: Figure S5a). Besides, to check if there is any other critical transition that leads to different survival time, a series of survival analysis has been carried out. As shown in Additional file 1: Figure S5b-S5c, statistically there is little difference $(p=0.4741$; Additional file 1: Figure S5b) between the survival time of stages-IA samples and that of stage-IB samples, and little statistical differences ( $p=0.5671$; Additional file 1: Figure $55 c$ ) in survival time among samples from stages
IIB, IIIA, IIIB, IV. In other word, there is no other critical transition point in either before-transition period (stages IA-IB), or after-transition period (stages IIB-IV). These results demonstrate that given high-throughput molecular data, the critical transition associated with disease deterioration and survival time in LUSC can be identified by sKLD.

In addition, at the identified critical stage (stage IIA), the top 5\% most significant genes in terms of the cumulative area of the case sample are selected as "sKLD-signaling genes" for further functional analysis. Some genes in the common "sKLD-signaling genes" have been reported to be associated with the process of LUSC (Table 1). For instance, the miR-195 axis regulates lung squamous cell carcinoma (LUSC) progression through 
Table 1 The genes with high frequency in 13 "sKLD-signaling genes" groups in the critical stage (stage IIA) for LUSC

\begin{tabular}{|c|c|c|c|c|}
\hline Gene & Frequency & Location & Family* & Relation with cancer progression \\
\hline BIRC5 & 13 & Cytoplasm & other & The miR-195 axis regulates lung squamous cell carcinoma (LUSC) progression through BIRC [39]. \\
\hline CCNA2 & 13 & Nucleus & other & $\begin{array}{l}\text { CCNA2 promotes invasion and migration of non-small cell lung cancer cells through integrin av } \beta 3 \\
\text { signaling } \\
\text { pathway [40]. }\end{array}$ \\
\hline CDC6 & 13 & Nucleus & other & $\begin{array}{l}\text { miR-26a/b inhibits directly migration, invasion, and proliferation of lung cancer cells by targeting } \\
\text { CDC6 [41]. }\end{array}$ \\
\hline CKS1B & 13 & Other & kinase & $\begin{array}{l}\text { CKS1B is a lung cancer-related gene, knockdown of which can result in a significant decrease in } \\
\text { lung cancer cell proliferation, invasion and migration [42]. }\end{array}$ \\
\hline E2F8 & 13 & Nucleus & $\begin{array}{l}\text { transcription } \\
\text { regulator }\end{array}$ & $\begin{array}{l}\text { Depletion of E2F8 inhibits cell proliferation and tumor growth in lung cancer, thus E2F8 can be } \\
\text { considered as a novel therapeutic target for lung cancer [43]. }\end{array}$ \\
\hline FOXM1 & 13 & Nucleus & $\begin{array}{l}\text { transcription } \\
\text { regulator }\end{array}$ & $\begin{array}{l}\text { Knockdown of FOXM1 inhibits the cell proliferation } \\
\text { in LUSC [44]. }\end{array}$ \\
\hline ITPKA & 13 & Cytoplasm & kinase & $\begin{array}{l}\text { ITPKA serves as an early diagnostic marker in lung cancer, whose overexpression promotes } \\
\text { tumorigenesis [45]. }\end{array}$ \\
\hline MCM2 & 13 & Nucleus & enzyme & $\begin{array}{l}\text { MCM2 regulates proliferation and cell cycle in lung squamous cell carcinoma, whose overexpressed } \\
\text { protein is obviously associated with malign differentiated degree and lymph node metastasis [46]. }\end{array}$ \\
\hline
\end{tabular}

BIRC5 [39]. CCNA2 promotes invasion and migration of non-small cell lung cancer cells through integrin $\alpha v \beta 3$ signaling pathway [40]. miR-26a/b inhibits directly migration, invasion, and proliferation of lung cancer cells by targeting CDC6 [41]. CKS1B is a lung cancer-related gene, knockdown of which results in a significant decrease in lung cancer cell proliferation, invasion and migration [42]. Depletion of E2F8 inhibits cell proliferation and tumor growth in lung cancer, thus E2F8 can be considered as a novel therapeutic target for lung cancer [43]. Knockdown of FOXM1 inhibits the cell proliferation in LUSC [44]. ITPKA serves as an early diagnostic marker in lung cancer, whose overexpression promotes tumorigenesis [45]. MCM2 regulates proliferation and cell cycle in lung squamous cell carcinoma, whose overexpressed protein is obviously associated with malign differentiated degree and lymph node metastasis [46]. The sKLD-signaling genes for the five tumor datasets were provided in Additional file 2.

Besides, functional enrichment through $\mathrm{GO}$ analysis shows that the common sKLD-signaling genes are involved in the biological processes including cytoskeleton organization, chromosome condensation, regulation of cell division and others (Table 2). These biological processes are associated with the progression of cancer. Furthermore, through IPA (Ingenuity Pathway Analysis), these common genes are also enriched to cancer-related function annotation, such as lung squamous cell carcinoma, development of malignant tumor and lung carcinoma (Table 2).

\section{The critical state of LUAD}

In Fig. 4b, the peak of the SKLD score at stage IIB suggests that there is a critical transition of LUAD after stage IIB. References showed that after stage IIB, ipsilateral mediastinal or subcarinal lymph nodes were metastasized (stage IIIA) and tumor began to invade heart, great vessels and trachea (stages IIIA-IIIB) [47]. As shown in Fig. $4 \mathrm{~g}$, there is significant difference ( $p=3 \mathrm{E}$ 07) between the survival curves of samples before and after stage IIB of LUAD. Clearly, the survival time of before-transition samples (samples from stages IA-IIB) is considerably longer than that of after-transition samples (samples from stages IIIA-IV). For the samples solely from two stages IIB and IIIA around the critical transition point, the survival time of stage-IIB samples is

Table 2 The functional enrichment of common "sKLD-signaling genes" in the critical stage samples for LUSC

\begin{tabular}{lllll}
\hline Gene Ontology Consortium & & & IPA \\
\cline { 5 - 6 } enriched biological process & enriched $p$ value & & enriched biological process & enriched $p$ value \\
\hline cytoskeleton organization (GO:0007010) & $2.00 \mathrm{E}-12$ & & mitosis of tumor cell lines & $1.39 \mathrm{E}-39$ \\
chromosome condensation (GO:0030261) & $4.49 \mathrm{E}-09$ & & development of malignant tumor & $6.37 \mathrm{E}-29$ \\
regulation of cell division (GO:0051302) & $1.23 \mathrm{E}-08$ & lung squamous cell carcinoma & $1.62 \mathrm{E}-24$ \\
sister chromatid cohesion (GO:0007062) & $2.43 \mathrm{E}-06$ & $1.03 \mathrm{E}-04$ & lung carcinoma & $7.28 \mathrm{E}-22$ \\
isotype switching (GO:0045190) & $6.57 \mathrm{E}-04$ & respiratory system tumor & $1.19 \mathrm{E}-19$ \\
programmed cell death (GO:0012501) & & lymphocytic neoplasm & $9.89 \mathrm{E}-12$ \\
\hline
\end{tabular}


much longer than stage-IIIA samples $(p=0.012$; Additional file 1: Figure S5d). Besides, statistically it shows little significant difference ( $p=0.4421$; Additional file 1: Figure S5e) among the survival curves of samples from stages IA, IB, IIA (the stages before the critical state), and little difference ( $p=0.1649$; Additional file 1: Figure S5f) among the survival curves of samples from stages IIIA, IIIB, IV (the stages after the critical state), which show that stage IIB of LUAD is highly associated with the critical transition of survival time.

The functional analysis is carried out based on "sKLDsignaling genes". Through literature searching, some genes in the common "sKLD-signaling genes" have been shown to be associated with the process of LUAD (Table 3). For example, PYCR1 may be a novel therapeutic target for inhibiting cell proliferation in lung cancer [48]. ETV4 promotes proliferation and invasion of lung adenocarcinoma by transcriptionally upregulating MSI2 [49]. Knockdown of PITX2 inhibits cell proliferation, migration and invasion of LUAD [50]. MDK plays an important role in non-small cell lung cancer progression and prognosis and may act as a convincing prognostic indicator for non-small cell lung cancer patients [51]. Blocking glutamine-mediated induction of PPAT inhibits cell proliferation and invasion in LUAD [52]. TOP2A is an ideal candidate as miR-144-3p target in non-small cell lung cancer, while MiR-144-3p expression is significantly correlated with lymph node metastasis and vascular invasion [53]. HOXC13 promotes proliferation of lung adenocarcinoma via modulation of CCND1 and CCNE1 [54]. Up-regulation of SRPK1 in non-small cell lung cancer promotes the growth and migration of cancer cells [55].
Moreover, functional enrichment through GO analysis shows that the common "sKLD-signaling genes" are involved in the biological processes of meiotic cell cycle, cell cycle checkpoint, cytokinesis, and so on (Table 4). These biological processes are associated with the progression of cancer. In addition, these common genes were also related to lung adenocarcinoma and development of lung tumor by functional enrichment in IPA (Table 4).

\section{The critical state of STAD}

For STAD, as shown in Fig. 4c, the drastic transitions of average sKLD appeared in stage IIIB, which indicated the imminent critical transition at stage IV. According to the division of clinical stages of STAD, the deterioration into stage IV means an advanced metastatic stage, in which the tumor has spread to nearby tissues or metastasized to other parts of the human body [56]. Fig. 4h shows that there is significantly difference $(p=0.0257)$ between the survival time of two group of samples, i.e., samples respectively from the before-transition period (stages IA-IIIB) and from the after-transition period (stages IV) of STAD. It is also noted that the survival time of samples from stage IIIB is significantly longer than that from stage IV $(p=0.0215$; Additional file 1: Figure S5 g). Besides, there is little significant difference $(p=0.1252$; Additional file 1: Figure S5h) among survival curves of samples from the period prior to the critical transition, i.e., stages IA-IIIA. These results demonstrate that the sKLD detected the early-warning signals of a critical transition of survive time and distant metastasis at stage IV.

Table 3 The genes with high frequency in 59 "sKLD-signaling genes" groups in the critical stage (stage IIB) for LUAD

\begin{tabular}{|c|c|c|c|c|}
\hline Gene & Frequency & Location & Family* & Relation with cancer progression \\
\hline PYCR1 & 54 & Cytoplasm & enzyme & PYCR1 may be a novel therapeutic target for inhibiting cell proliferation in lung cancer [48]. \\
\hline ETV4 & 50 & Nucleus & $\begin{array}{l}\text { transcription } \\
\text { regulator }\end{array}$ & $\begin{array}{l}\text { ETV4 promotes proliferation and invasion of lung adenocarcinoma by transcriptionally } \\
\text { upregulating } \\
\text { MSI2 [49]. }\end{array}$ \\
\hline PITX2 & 50 & Nucleus & $\begin{array}{l}\text { transcription } \\
\text { regulator }\end{array}$ & Knockdown of PITX2 inhibits cell proliferation, migration and invasion of LUAD [50]. \\
\hline MDK & 49 & $\begin{array}{l}\text { Extracellular } \\
\text { Space }\end{array}$ & growth factor & $\begin{array}{l}\text { MDK plays an important role in non-small cell lung cancer progression and prognosis and may } \\
\text { act as a convincing prognostic indicator for non-small cell lung cancer patients [51]. }\end{array}$ \\
\hline PPAT & 49 & Cytoplasm & enzyme & $\begin{array}{l}\text { Blocking glutamine-mediated induction of PPAT } \\
\text { inhibits cell proliferation and invasion in } \\
\text { LUAD [52]. }\end{array}$ \\
\hline TOP2A & 49 & Nucleus & enzyme & $\begin{array}{l}\text { TOP2A is an ideal candidate for miR-144-3p targets in non-small cell lung cancer and MiR-144- } \\
3 p \text { expression is significantly correlated with stage, lymph node metastasis and vascular invasion } \\
\text { [53]. }\end{array}$ \\
\hline $\mathrm{HOXC13}$ & 48 & Nucleus & $\begin{array}{l}\text { transcription } \\
\text { regulator }\end{array}$ & $\begin{array}{l}\text { HOXC13 promotes proliferation of lung adenocarcinoma via modulation of CCND1 and CCNE1 } \\
\text { [54]. }\end{array}$ \\
\hline SRPK1 & 48 & Nucleus & kinase & $\begin{array}{l}\text { Up-regulation of SRPK1 in non-small cell lung cancer can promote the growth and migration of } \\
\text { cancer } \\
\text { cells [55]. }\end{array}$ \\
\hline
\end{tabular}


Table 4 The functional enrichment of common "sKLD-signaling genes" in the critical stage samples for LUAD

\begin{tabular}{|c|c|c|c|}
\hline \multicolumn{2}{|l|}{ Gene Ontology Consortium } & \multicolumn{2}{|l|}{ IPA } \\
\hline enriched biological process & enriched $p$ value & enriched biological process & enriched $p$ value \\
\hline cell cycle checkpoint (GO:0000075) & $1.92 \mathrm{E}-15$ & Lung cancer & 7.77E-28 \\
\hline meiotic cell cycle (GO:0051321) & $1.12 \mathrm{E}-11$ & Lung tumor & 2.97E-27 \\
\hline cytokinesis (GO:0000910) & $1.71 \mathrm{E}-06$ & Lung carcinoma & $1.42 \mathrm{E}-23$ \\
\hline fucosylation (GO:0036065) & $1.65 \mathrm{E}-04$ & Non-small cell lung carcinoma & $6.74 \mathrm{E}-22$ \\
\hline regulation of cell development (GO:0060284) & 3.15E-04 & Development of lung tumor & $1.48 \mathrm{E}-21$ \\
\hline regulation of developmental process (G0:0050793) & $9.30 \mathrm{E}-04$ & Lung adenocarcinoma & $1.85 \mathrm{E}-21$ \\
\hline
\end{tabular}

Some "sKLD-signaling genes" have been found in literatures and identified to be associated with the process of STAD (Table 5). For instance, COL10A1 promotes invasion and metastasis in gastric cancer through transcriptional regulation of SOX9 and the involvement of the TGF- $\beta$ signaling pathway [57]. BGN promotes tumor invasion and metastasis of gastric cancer both in vitro and in vivo [58]. CTHRC1 may be associated with metastasis in human gastric cancer [59]. Let-7b inhibits cell proliferation, migration, and invasion through targeting CTHRC1 in gastric cancer [60]. Enforced expression of SALL4 not only enhances the proliferation and migration of human gastric cancer cells, but promotes the growth and metastasis of gastric xenograft tumor in vivo [61]. Knockdown of MMP11 inhibits proliferation and invasion of gastric cancer cells [62]. The overexpression of MAP4K4 promotes cancer progression or metastasis [63, 64]. MiR-211 inhibits cell proliferation and invasion of gastric cancer by down-regulating SOX4 [65]. The overexpression of FOXS1 in gastric cancer cell lines can inhibit proliferation, metastasis and epithelial-mesenchymal transition of tumor through downregulating wnt/ $\beta$-catenin pathway [66].
Besides, based on GO analysis, the common "sKLD-signaling genes" are enriched into the biological processes associated with the progression of cancer, e.g., extracellular matrix organization, collagen fibril organization and ribosome biogenesis (Table 6). Furthermore, according to IPA, the common "sKLD-signaling genes" are also enriched to cancerrelated function annotation including digestive organ tumor, digestive system cancer and abdominal cancer (Table 6). The common sKLD-signaling genes for LUSC, LUAD and STAD were provided in Additional file 4.

\section{The critical state of THCA}

As shown in Fig. 4d, for THCA, the sKLD score reaches its peak at stage II, signaling the imminent critical transition at stage III. There was extension to sternothyroid muscle or perithyroid soft tissues and regional lymph node metastasis in stage III [67]. There is significant difference between the survival curves before and after stage II in THCA samples $(p=0)$ (Fig. 4i). It is seen that the survival times of samples before the critical state were significantly longer than for samples after the critical state. There was no significant difference in survival curves among samples in stages III, IV

Table 5 The genes with high frequency in 20 "sKLD-signaling genes" groups in the critical stage (stage IIIB) for STAD

\begin{tabular}{|c|c|c|c|c|}
\hline Gene & Frequency & Location & Family* & Relation with cancer progression \\
\hline COL10A1 & 19 & $\begin{array}{l}\text { Extracellular } \\
\text { Space }\end{array}$ & other & $\begin{array}{l}\text { COL10A1 promotes invasion and metastasis in gastric cancer through transcriptional regulation } \\
\text { of SOX9 and the involvement of the TGF- } \beta \text { signaling pathway [57]. }\end{array}$ \\
\hline BGN & 18 & $\begin{array}{l}\text { Extracellular } \\
\text { Space }\end{array}$ & other & BGN promote tumor invasion and metastasis of gastric cancer both in vitro and in vivo [58]. \\
\hline CTHRC1 & 18 & $\begin{array}{l}\text { Extracellular } \\
\text { Space }\end{array}$ & other & $\begin{array}{l}\text { CTHRC1 may be associated with metastasis in human gastric cancer [59]. Let-7b inhibits cell } \\
\text { proliferation, migration, and invasion through targeting CTHRC1 in gastric cancer [60]. }\end{array}$ \\
\hline SALL4 & 18 & Nucleus & $\begin{array}{l}\text { transcription } \\
\text { regulator }\end{array}$ & $\begin{array}{l}\text { Enforced expression of SALL4 not only enhances the proliferation and migration of human } \\
\text { gastric cancer cells, but promotes the growth and metastasis of gastric xenograft tumor in vivo } \\
\text { [61]. }\end{array}$ \\
\hline MMP11 & 18 & $\begin{array}{l}\text { Extracellular } \\
\text { Space }\end{array}$ & peptidase & Knockdown of MMP11 inhibits proliferation and invasion of gastric cancer cells [62]. \\
\hline MAP4K4 & 18 & Cytoplasm & kinase & MAP4K4 overexpression promotes cancer progression or metastasis $[63,64]$. \\
\hline SOX4 & 18 & Nucleus & $\begin{array}{l}\text { transcription } \\
\text { regulator }\end{array}$ & MiR-211 inhibits cell proliferation and invasion of gastric cancer by down-regulating SOX4 [65]. \\
\hline FOXS1 & 17 & Nucleus & $\begin{array}{l}\text { transcription } \\
\text { regulator }\end{array}$ & $\begin{array}{l}\text { Overexpression of FOXS1 in gastric cancer cell lines can inhibit proliferation, metastasis, and } \\
\text { epithelial-mesenchymal transition of tumor through downregulating wnt/ } \beta \text {-catenin pathway } \\
\text { [66]. }\end{array}$ \\
\hline
\end{tabular}


Table 6 The functional enrichment of common "sKLD-signaling genes" in the critical stage samples for STAD

\begin{tabular}{|c|c|c|c|}
\hline \multicolumn{2}{|l|}{ Gene Ontology Consortium } & \multicolumn{2}{|l|}{ IPA } \\
\hline enriched biological process & enriched $p$ value & enriched biological process & enriched $p$ value \\
\hline extracellular matrix organization (GO:0030198) & 3.32E-14 & digestive organ tumor & 4.04E-51 \\
\hline collagen fibril organization (GO:0030199) & $5.31 \mathrm{E}-13$ & digestive system cancer & $1.63 \mathrm{E}-50$ \\
\hline ribosome biogenesis (GO:0042254) & $1.08 \mathrm{E}-09$ & abdominal cancer & 2.07E-49 \\
\hline regulation of cell cycle (GO:0051726) & 1.61E-08 & gastrointestinal tract cancer & $7.11 \mathrm{E}-45$ \\
\hline collagen metabolic process (GO:0032963) & 3.67E-05 & gastrointestinal carcinoma & 1.49E-44 \\
\hline regulation of protein ubiquitination (GO:0031396) & 9.60E-05 & development of digestive organ tumor & $5.9 \mathrm{E}-27$ \\
\hline
\end{tabular}

(the stages after the critical state) ( $p=0.5158$; Additional file 1: Figure S5i). The survival times of samples in stage II were significantly longer than for samples in stage III $(p=$ 0.0381; Additional file 1: Figure S5j). These results illustrate the sKLD can detect the early-warning signals associated with disease deterioration in THCA. Furthermore, the functional analyses of some "sKLD-signaling genes" are performed through IPA and literature searching, which is provided in Additional file 1: Table S2. The enrichment analysis for the common "sKLD-signaling genes" is carried out based on GO and IPA analysis, which is given in Additional file 1: Table S3.

\section{The critical state of COAD}

For COAD, the drastic increase of the sKLD score from stage I to stage II suggests a critical deterioration after stage II (Fig. 4e). There are lymph nodes metastasis and tumor directly invade other organs or structures in stage III [68]. There was significant difference between the survival curves before and after stage II in COAD samples $(p=0)$ (Fig. 4j). As shown in Fig. 4j, the survival time of samples before the critical state were obviously longer than that of samples after the critical state. There were no statistics difference in survival curves among samples in stages III, IV (the stages after the critical state) ( $p=0.1048$; Additional file 1: Figure S5k). The survival times of samples in stage II were significantly longer than for samples in stage III $(p=0.0067$; Additional file 1: Figure S5 1). These results demonstrate the sKLD can provide the early-warning signals associated with disease deterioration in COAD. Moreover, functional analyses of some "sKLD-signaling genes" are performed through IPA and literature searching, which is given in Additional file 1: Table S4. The enrichment analysis for the common "sKLD-signaling genes" is performed through GO and IPA analysis, which is provided in Additional file 1: Table S5.

\section{Discussion}

Detecting the early-warning signal for the sudden deterioration is crucial to most complex diseases. However, it is a challenging task to identify the pre-disease state prior to the occurrence of obvious symptoms due to the lack of samples, that is, there is usually only one single sample for an individual at a time point before an accurate diagnosis is made. Clearly, such singlesample problem, rising from clinical and experimental practice, leads to the failure of traditional statistic method, and thus requires new approaches that help to overcome the sample limitation. In this study, we proposed a single-sample-based computational framework, the sKLD method, to quantify the disturbance on the background caused by a sample. The sKLD has been applied to real-world datasets and successfully identifies the tipping points or critical states of complex diseases. Specifically, the significant change of sKLD score indicates the pre-disease state of phosgene-induced acute lung injury before the deterioration into pulmonary edema, the critical stage of (stage IIA) of LUSC prior to the lymph nodes metastasis, the critical stage (stage IIB) of LUAD before lymph nodes were metastasized, the critical stage (stage IIIB) of STAD before distant metastasis, the critical stage (stage II) of THCA before lymph node metastasis, and the critical stage (stage II) of THCA before lymph node metastasis. All these identified critical stages were validated by the survival analysis, that is, the patient would have a significantly better prognosis if they were diagnosed before the critical stage. Besides, at any other stages, there was no significant difference in the prognosis, suggesting that the identified critical stage is accurate and closely associated with prognosis. The functional analysis of sKLDsignaling genes is consistent with the upcoming deteriorations of diseases.

There are three advantages of the proposed method. First, in contrast to the traditional biomarkers that are used to "diagnose disease" based on the information of differential expressions, sKLD is capable to "predict disease" based on the information of differential distributions among biomolecules. Second, given some reference samples, sKLD works with only a single sample. Third, it should be noted that sKLD is a model-free method, which implies that in the sKLD strategy there is neither feature selection nor model/parameter training 
procedure. It is thus different from the traditional machine learning or classification methods which, to produce a robust model in the learning process, requires a substantial number of case and control samples to avoid the overfitting problem.

\section{Conclusions}

We proposed a novel computational method sKLD solely based on a single case sample. This method can effectively detect the pre-disease state of complex diseases, a state with high susceptibility before the disease onset or deterioration. As the algorithm shown in Methods section, the sKLD is easy to implement and very flexible. It is therefore of great potential in personalized pre-disease diagnosis and prevention medicine. The identification of sKLD-signaling genes is also helpful in elucidating molecular mechanism of disease progression, and discovering prognosis indicators.

\section{Methods}

\section{Theoretical background}

The theoretical background is our recently proposed DNB theory. Specifically, in order to theoretically and mathematically describe the dynamics of a complex disease, its evolution is usually modeled as a timedependent nonlinear dynamical system [23, 69], in which the sudden deterioration is regarded as a state transition at a bifurcation point [16]. In ideal situation with small noise, when a complex system is near the critical point, among all observed variables there exists a dominant group defined as the DNB biomolecules, which satisfy the following three conditions based on the observed data [10]:

- The correlation $\left(\mathrm{PCC}_{\text {in }}\right)$ between any pair of members in the DNB group rapidly increases;

- The correlation ( $\mathrm{PCC}_{\text {out }}$ ) between one member of the DNB group and any other non-DNB member rapidly decreases;

- The standard deviation $\left(\mathrm{SD}_{\mathrm{in}}\right)$ or coefficient of variation for any member in the DNB group drastically increases.

The above three properties are necessary conditions of the state transition at a codimension-one bifurcation point, and can also be approximately stated as: the occurrence of a group of biomolecules whose expressions are strongly fluctuating and highly correlated, implies an upcoming critical transition. These three properties are the theoretical basis of DNB method and have been proved in the supplementary information of our previous work [10].

From the above three properties, it is clear that the critical transition of a system is actually indicated by "the transition of distribution", that is, for some variables (DNB members), their distribution would significantly change when the system approaches the critical transition point. Therefore, by exploring the differential distributions (rather than differential expressions) of some variables, it is possible to predict the upcoming qualitative state transition. On the other hand, a sample of high-throughput data enables us to analyze the expressions of thousands of genes simultaneously. Such a high-dimensional sample is actually enriched with dynamic information of accumulated effects, such as the gene interaction after a long-term development of the concerned biological system.

The Kullback-Leibler divergence (K-L divergence) was widely employed to measure the difference between two data distributions [70]. It provides a theoretical basis for data differencing [71], outlier detection [72] and evaluating sample similarity [73, 74]. Between two distributions $P$ and $Q$, the $\mathrm{K}-\mathrm{L}$ divergence is defined as

$$
D_{K L}(P, Q)=\sum_{\mathrm{k}} \ln \left(\frac{P(k)}{Q(k)}\right) P(k) .
$$

It should be noted that the K-L divergence in Eq. (1) is actually not a true metric, but usually serves as a measure of the similarity between distributions $P$ and $Q$. Particularly, $D_{K L}(P, Q)$ is zero only when the distribution $P$ is identical with the distribution $Q . D_{K L}(P, Q)$ is positive when the distribution $P$ is different from $Q$. Clearly, for the original K-L divergence, there is $D_{K L}(P, Q) \neq D_{K L}(Q$, $P)$. In this study, we use a symmetric measure defined as

$D_{K L}(P, Q)=\frac{\sum_{\mathrm{k}} \ln \left(\frac{P(k)}{Q(k)}\right) P(k)+\sum_{\mathrm{k}} \ln \left(\frac{Q(k)}{P(k)}\right) Q(k)}{2}$.

\section{Algorithm to identify the tipping point based on SKLD}

Regarding a biological system as a time-dependent nonlinear dynamical system with $m$ genes/variables, then at each time point, the state of such system is expressed by a high-dimensional vector, i.e., the expressions of $m$ genes/variables. A computational way is then developed to exploring the dynamic difference between the normal state and pre-disease state.

Given a set of reference samples (samples from normal cohort which are used as the background that represent the healthy or relatively healthy individuals), the following algorithm is proposed to identify the pre-disease state by using only one case sample.

[Step 1] Prepare a set of reference samples. The samples derived from the normal cohort are regarded as reference samples, which represent the background of relatively healthy individuals in the normal state. For numerical simulation, samples from a few initial time points are viewed as reference/normal samples. For real 
datasets, samples from a normal cohort or normal tissue are chosen as reference/normal samples, e.g., for the stage-course data from TCGA, the tumor-adjacent samples are taken as the reference.

[Step 2] Fit a distribution for each gene in terms of the expressions from the reference samples. Specifically, for a gene $g_{i}$, a Gaussian distribution $\mathrm{D}_{\mathrm{g}_{\mathrm{i}}}$ is fitted based on the $k$ expressions of $g_{i}$ in the reference samples $\left\{S_{1}, S_{2}\right.$, ..., $\left.S_{\mathrm{k}}\right\}$. Then, a $k$-dimensional vector $\left(\operatorname{area}\left(\mathrm{D}_{\mathrm{g}_{\mathrm{i}}}\left(S_{1}\right)\right)\right.$, area $\left(\mathrm{D}_{\mathrm{g}_{\mathrm{i}}}\left(S_{2}\right)\right), \ldots$, area $\left.\left(\mathrm{D}_{\mathrm{g}_{\mathrm{i}}}\left(S_{k}\right)\right)\right)$ is obtained, in which the $j$-th element is the cumulative area (its definition was shown in the Eq. (S4) of Additional file 1) determined by the fitted distribution $\mathrm{D}_{\mathrm{g}_{\mathrm{i}}}$ and the expression of $\mathrm{g}_{i}$ in the $j$-th sample $S_{j}$ (Fig. 1b).

[Step 3] Construct the reference distribution $P$ as follows.

$$
\begin{aligned}
& P=\left[\begin{array}{c}
p_{1} \\
p_{2} \\
\vdots \\
p_{m}
\end{array}\right], p_{i}
\end{aligned}
$$

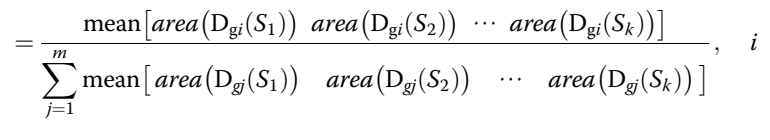

$$
\begin{aligned}
& =1,2, \ldots, m \text {. }
\end{aligned}
$$

[Step 4] For a single case sample $\mathrm{s}_{\text {case }}$ of an individual, construct a perturbed distribution $Q$ based on $\mathrm{s}_{\text {case }}$ as follows.

$$
\begin{aligned}
Q & =\left[\begin{array}{c}
q_{1} \\
q_{2} \\
\vdots \\
q_{m}
\end{array}\right], q_{i}=\frac{\operatorname{area}\left(\mathrm{D}_{\mathrm{g} i}\left(S_{\text {case }}\right)\right)}{\sum_{j=1}^{m} \operatorname{area}\left(D_{g j}\left(S_{\text {case }}\right)\right)}, i \\
& =1,2, \ldots, m .
\end{aligned}
$$

For both distributions $P$ and $Q$, it is clear that $\sum_{j=1}^{m} p_{j}=1$ and $\sum_{j=1}^{m} q_{j}=1$.

[Step 5] Calculate the sKLD score based on Eq. (2). Clearly, such score evaluated the difference between the reference distribution $P$ and the perturbed distribution $Q$.

According to the DNB theory, when the system approaches the critical state, the DNB biomolecules exhibit significantly collective behaviors with fluctuations (see the supplementary information of reference [18] for detailed derivation in the ideal situation), which leads to that the distributions of DNB genes in a pre-disease state are different from those in a normal state. Thus, the background distribution from a set of reference/normal samples significantly distinct to the perturbed distribution from a new case sample, leading to the increase of
sKLD score in Eq. (2). Thus, sKLD score can provide the early-warning signals of the critical transition. From above algorithm, it is seen that the proposed method is data-driven, and thus model free.

\section{Data processing and functional analysis}

The proposed method has been applied to six real datasets, i.e., the time-course dataset GSE2565 from NCBI GEO database (http://www.ncbi.nlm.nih.gov/geo) and five stage-course datasets LUSC, LUAD, STAD, THCA and COAD from TCGA database (http://cancergenome. nih.gov). The omics dataset GSE2565 comprises expression profiles from a mouse experiment, in which pulmonary edema was triggered by inhalation of carbonyl chloride. In this dataset, we discarded the probes without corresponding NCBI Entrez gene symbol. For each gene mapped by multiple probes, the average value was employed as the gene expression. The five stage-course datasets from TCGA contained RNA-Seq data and included both tumor and tumor-adjacent samples. The tumor samples were divided into different stages based on clinical (stage) information from TCGA, and the samples without stage information were ignored.

For all the diseases, functional annotations were performed by searching the NCBI gene database (http:// www.ncbi.nlm.nih.gov/gene). The enrichment analyses were separately obtained using web service tools from the Gene Ontology Consortium (GOC, http://geneontology.org) and client software from Ingenuity Pathway Analysis (IPA, http://www.ingenuity.com/products/ipa).

\section{Supplementary information}

Supplementary information accompanies this paper at https://doi.org/10. 1186/s12864-020-6490-7.

Additional file 1. Identifying critical state by single-sample Kullback-Leibler divergence.

Additional file 2. The signaling genes of LUSC, LUAD, STAD, THCA and COAD.

Additional file 3. The common signaling genes for acute lung injury. Additional file 4. The common sKLD-signaling genes for LUSC, LUAD and STAD

\section{Abbreviations}

COAD: Colon adenocarcinoma; DNB: The dynamical network biomarker; LUAD: Lung adenocarcinoma; LUSC: Squamous cell carcinoma; PCC: Pearson correlation coefficient; SD: Standard deviation; sKLD: Single-sample KullbackLeibler divergence; STAD: Stomach adenocarcinoma; TCGA: The cancer genome atlas; THCA: Thyroid carcinoma

\section{Acknowledgements}

Not applicable.

Authors' contributions

$\mathrm{RL}$ and $\mathrm{PC}$ conceived the research. JYZ performed the numerical simulation and real data analysis. All authors wrote the paper. All authors read and approved the final manuscript. 


\section{Funding}

This work was supported by National Natural Science Foundation of China (Nos. 11771152, 11901203, 11971176, 31930022), Guangdong Basic and Applied Basic Research Foundation (2019B151502062), China Postdoctoral Science Foundation funded project (No. 2019M662895) and the Fundamental Research Funds for the Central Universities (2019MS111).

\section{Availability of data and materials}

Lung squamous cell carcinoma (LUSC), lung adenocarcinoma (LUAD), stomach adenocarcinoma (STAD), thyroid carcinoma (THCA) and colon adenocarcinoma (COAD) are available from the cancer genome atlas (TCGA) database (http:// cancergenome.nih.gov). Acute lung injury (GSE2565) is available from NCBI GEO database (http://www.ncbi.nlm.nih.gov/geo). The source code of algorithm is accessed in (https://github.com/zhongjiayuna/KL_Project).

\section{Ethics approval and consent to participate} Not applicable.

\section{Consent for publication}

Not applicable.

\section{Competing interests}

The authors declare that they have no competing interests.

Received: 14 October 2019 Accepted: 13 January 2020

Published online: 28 January 2020

\section{References}

1. Bestelmeyer BT, Ellison AM, Fraser WR, Gorman KB, Holbrook SJ, Laney CM, et al. Analysis of abrupt transitions in ecological systems. Thermochim Acta. 2001;403(1):137-51. https://doi.org/10.1890/ES11-00216.1.

2. Corrado R, Cherubini AM, Pennetta C. Early warning signals of desertification transitions in semiarid ecosystems. Phys Rev E. 2014;90(6): 062705

3. Lenton TM, Myerscough RJ, Marsh R, Livina VN, Price AR, Cox SJ, et al. Using GENIE to study a tipping point in the climate system. Philos Trans R Soc A Math Phys Eng Sci. 2008;367(1890):871-84. https://doi.org/10.1098/rsta.2008.0171.

4. Boulton, C. A., Allison, L. C., \& Lenton, T. M. (2014). Early warning signals of Atlantic Meridional overturning circulation collapse in a fully coupled climate model. Nat Commun. 2014; 5: 5752

5. Liu R, Chen P, Aihara K, Chen L. Identifying early-warning signals of critical transitions with strong noise by dynamical network markers. Sci Rep. 2015 5(1):17501-13. https://doi.org/10.1038/srep17501.

6. Drehmann M, Juselius M. Evaluating early warning indicators of banking crises: satisfying policy requirements. Int J Forecasting. 2014;30(3):759-80

7. Veraart AJ, Faassen EJ, Dakos V, van Nes EH, Lürling M, Scheffer M. Recovery rates reflect distance to a tipping point in a living system. Nat. 2012 481(7381):357. https://doi.org/10.1038/nature10723.

8. van de Leemput IA, Wichers $M$, Cramer AO, Borsboom D, Tuerlinckx F, Kuppens P, van Nes EH, Viechtbauer W, Giltay EJ, Aggen SH, Derom C. Critical slowing down as early warning for the onset and termination of depression. Pro Nat Acad Sci. 2014;111(1):87-92.

9. Scarpino SV, Petri G. On the predictability of infectious disease outbreaks. Nat Commun. 2019:10(1):898

10. Chen L, Liu R, Liu ZP, Li M, Aihara K. Detecting early-warning signals for sudden deterioration of complex diseases by dynamical network biomarkers. Sci Rep. 2012;2(1):342-9. https://doi.org/10.1038/srep00342.

11. Chen P, Liu R, Aihara K, Chen L. Identifying critical differentiation state of MCF-7 cells for breast cancer by dynamical network biomarkers. Front Genet. 2015;6:252. https://doi.org/10.3389/fgene.2015.00252.

12. Venegas JG, Winkler T, Musch G, Melo MFV, Layfield D, Tgavalekos N, et al. Self-organized patchiness in asthma as a prelude to catastrophic shifts. Nat. 2005:434(7034):777-82. https://doi.org/10.1038/nature03490.

13. Litt B, Esteller R, Echauz J, D'Alessandro M, Shor R, Henry T, et al. Epileptic seizures may begin hours in advance of clinical onset: a report of five patients. In Applications of Intelligent Control to Engineering Systems. Springer Dordrecht. 2009;225-245. doi:https://doi.org/10.1016/S0896-6273(01)00262-8.

14. Liu R, Li M, Liu ZP, Wu J, Chen L, Aihara K. Identifying critical transitions and their leading biomolecular networks in complex diseases. Sci Rep. 2012;2(1): 813-21. https://doi.org/10.1038/srep00813.
15. Strogatz SH, Friedman M, Mallinckrodt AJ, McKay S. Nonlinear dynamics and chaos: with applications to physics, biology, chemistry, and engineering. Comput Phys. 1994;8(5):532. https://doi.org/10.1063/1.2807947.

16. Scheffer M, Carpenter S, Foley JA, Folke C, Walker B. Catastrophic shifts in ecosystems. Nat. 2001;413(6856):591-6. https://doi.org/10.1038/35098000.

17. Liu R, Wang X, Aihara K, Chen L. Early diagnosis of complex diseases by molecular biomarkers, network biomarkers, and dynamical network biomarkers. Med Res Rev. 2014;34(3):455-78. https://doi.org/10.1002/med.21293.

18. Liu R, Yu X, Liu X, Xu D, Aihara K, Chen L. Identifying critical transitions of complex diseases based on a single sample. Bioinformatics. 2014;30(11): 1579-86. https://doi.org/10.1093/bioinformatics/btu084.

19. Liu R, Wang J, Ukai M, Sewon K, Chen P, Suzuki Y, et al. Hunt for the tipping point during endocrine resistance process in breast cancer by dynamic network biomarkers. J Mol Cell Biol. 2019;11(8):649-64. https://doi.org/10. 1093/jmcb/mjy059.

20. Richard A, Boullu L, Herbach U, Bonnafoux A, Morin V, Vallin E, et al. Singlecell-based analysis highlights a surge in cell-to-cell molecular variability preceding irreversible commitment in a differentiation process. PLoS Biol. 2016:14(12):e1002585. https://doi.org/10.1371/journal.pbio.1002585.

21. Lesterhuis WJ, Bosco A, Millward MJ, Small M, Nowak AK, Lake RA. Dynamic versus static biomarkers in cancer immune checkpoint blockade: unravelling complexity. Nat Rev Drug Discov. 2017;16(4):264 https://doi.org/10.1038/nrd.2016.233.

22. Chen P, Chen E, Chen L, Zhou XJ, Liu R. Detecting early-warning signals of influenza outbreak based on dynamic network marker. J Cell Mol Med. 2019;23(1):395-404. https://doi.org/10.1111/jcmm.13943.

23. Chen P, Li Y, Liu X, Liu R, Chen L. Detecting the tipping points in a threestate model of complex diseases by temporal differential networks. J Transl Med. 2017;15(1):217. https://doi.org/10.1186/s12967-017-1320-7.

24. Chen $P$, Liu R, Li Y, Chen L. Detecting critical state before phase transition of complex biological systems by hidden Markov model. Bioinformatics. 2016; 32(14):2143-50. https://doi.org/10.1093/bioinformatics/btw154.

25. Tan Z, Liu R, Zheng L, Hao S, Fu C, Li Z, et al. Cerebrospinal fluid protein dynamic driver network: at the crossroads of brain tumorigenesis. Methods. 2015;83:36-43. https://doi.org/10.1016/j. ymeth.2015.05.004

26. Liu R, Chen P, Chen L. Single-sample landscape entropy reveals the imminent phase transition during disease progression. Bioinformatics. 2019. https://doi.org/10.1093/bioinformatics/btz758.

27. Garcia-Ojalvo J, Elowitz MB, Strogatz SH. Modeling a synthetic multicellular clock: repressilators coupled by quorum sensing. Proc Nati Acad Sci. 2004; 101(30):10955-60. https://doi.org/10.1073/pnas.0307095101.

28. Sherman MS, Cohen BA. Thermodynamic state ensemble models of cisregulation. PLoS Comput Biol. 2012;8(3):e1002407. https://doi.org/10.1371/ journal.pcbi.1002407

29. Cantone I, Marucci L, lorio F, Ricci MA, Belcastro V, Bansal M. A yeast synthetic network for in vivo assessment of reverse-engineering and modeling approaches. Cell. 2009;137(1):172-81. https://doi.org/10.1016/j.cell. 2009.01.055

30. Chen Y, Kim JK, Hirning AJ, Josić K, Bennett MR. Emergent genetic oscillations in a synthetic microbial consortium. Sci. 2015;349(6251):986-9. https://doi.org/10.1126/science.aaa3794.

31. Li C, Chen L, Aihara K. Stability of genetic networks with SUM regulatory logic: Lur'e system and LMI approach. IEEE Trans Circuits Syst I: Regular Papers. 2006;53(11):2451-8. https://doi.org/10.1109/TCSI.2006.883882.

32. Gardner TS, Cantor CR, Collins JJ. Construction of a genetic toggle switch in Escherichia coli. Nat. 2000;403(6767):339-42. https://doi.org/10.1038/35002131.

33. O'Brien EL, Van Itallie E, Bennett MR. Modeling synthetic gene oscillators. Math Biosci. 2012;236(1):1-15. https://doi.org/10.1016/j.mbs.2012.01.001.

34. Sciuto AM, Phillips CS, Orzolek LD, Hege Al, Moran TS, Dillman JF. Genomic analysis of murine pulmonary tissue following carbonyl chloride inhalation. Chem Res Toxicol. 2005;18(11):1654-60. https://doi.org/10.1021/tx050126f.

35. Zeng T, Sun SY, Wang Y, Zhu H, Chen L. Network biomarkers reveal dysfunctional gene regulations during disease progression. FEBS J. 2013; 280(22):5682-95.

36. Li M, Zeng T, Liu R. \& Chen, L detecting tissue-specific early warning signals for complex diseases based on dynamical network biomarkers: study of type 2 diabetes by cross-tissue analysis. Brief Bioinformatics. 2013;15(2):229-43.

37. Dai H, Li L, Zeng T, Chen L. Cell-specific network constructed by single-cell RNA sequencing data. Nucleic Acids Res. 2019;47(11):e62.

38. Detterbeck FC, Postmus PE, Tanoue LT. The stage classification of lung cancer: diagnosis and management of lung cancer: American College of 
Chest Physicians evidence-based clinical practice guidelines. Chest. 2013; 143(5):e191S-210S. https://doi.org/10.1378/chest.12-2354.

39. Yu X, Zhang Y, Wu B, Kurie J M, Pertsemlidis A. The miR-195 axis regulates chemoresistance through TUBB and lung cancer progression through BIRC5. Mol Ther-Oncolytics. 2019;14:288-98.

40. Ruan JS, Zhou H, Yang L, Wang L, Jiang ZS, Wang SM. CCNA2 facilitates epithelial-to-mesenchymal transition via the integrin av $\beta 3$ signaling in NSCLC. Int J Clin Exp Pathol. 2017;10(8):8324-33.

41. Asghariazar V, Sakhinia E, Mansoori B, Mohammadi A, Baradaran B. Tumor suppressor microRNAs in lung cancer: An insight to signaling pathways and drug resistance. J Cell Biochem. 2019;120(12):19274-89. https://doi.org/10. 1002/jcb.29295.

42. Wang $L$, Zhao $H, X u Y$, Li J, Deng C, Deng Y. Systematic identification of lincRNA-based prognostic biomarkers by integrating lincRNA expression and copy number variation in lung adenocarcinoma. Int J Cancer. 2019; 144(7):1723-34. https://doi.org/10.1002/ijc.31865.

43. Park S A, Platt J, Lee J W, López-Giráldez F, Herbst R S, Koo J S. E2F8 as a novel therapeutic target for lung cancer. JNCl: J Natl Cancer Inst. 2015; 107(9):djv151. https://doi.org/10.1093/jnci/djv151.

44. Cheng Z, Yu C, Cui S, Wang H, lin H, Wang C, et al. circTP63 functions as a ceRNA to promote lung squamous cell carcinoma progression by upregulating FOXM1. Nat Commun. 2019;10(1):3200-12. https:/doi.org/10.1038/s41467-019-11162-4.

45. Wang YW, Ma X, Zhang YA, Wang MJ, Yatabe Y, Lam S, et al. ITPKA gene body methylation regulates gene expression and serves as an early diagnostic marker in lung and other cancers. J Thorac Oncol. 2016;1 (9): 1469-81. https://doi.org/10.1016/j.jtho.2016.05.010.

46. Wu W, Wang X, Shan C, Li Y, Li F. Minichromosome maintenance protein 2 correlates with the malignant status and regulates proliferation and cell cycle in lung squamous cell carcinoma. Onco Targets Ther. 2008;1 1:5025-34. https://doi.org/10.2147/OTT.S169002.

47. Goldstraw P, Crowley J, Chansky K, Giroux DJ, Groome PA, Rami-Porta R, et al. The IASLC lung Cancer staging project: proposals for the revision of the TNM stage groupings in the forthcoming (seventh) edition of the TNM classification of malignant tumours. J Thorac Oncol. 2007;2(8):706-14. https://doi.org/10.1097/JTO.0b013e31812f3c1a.

48. Cai F, Miao Y, Liu C, Wu T, Shen S, Su X, Shi Y. Pyrroline-5-carboxylate reductase 1 promotes proliferation and inhibits apoptosis in non-small cell lung cancer. Oncol Lett. 2018;15(1):731-40. https://doi.org/10.3892/ol.2017.7400.

49. Cheng T, Zhang Z, Cheng Y, Zhang J, Tang J, Tan Z, et al. ETV4 promotes proliferation and invasion of lung adenocarcinoma by transcriptionally upregulating MSI2. Biochem Biophys Res Commun. 2019;516(1):278-84. https://doi.org/10.1016/.jbbrc.2019.06.115.

50. Luo J, Yao Y, Ji S, Sun Q, Xu Y, Liu K, et al. PITX2 enhances progression of lung adenocarcinoma by transcriptionally regulating WNT3A and activating WNT/ß-catenin signaling pathway. Cancer Cell Int. 2019;19(1):96-110. https://doi.org/10.1186/s12935-019-0800-7.

51. Yuan K, Chen Z, Li W, Gao CE, Li G, Guo G, et al. MDK protein overexpression correlates with the malignant status and prognosis of nonsmall cell lung cancer. Arch Med Res. 2015;46(8):635-41. https://doi.org/10. 1016/j.arcmed.2015.11.006.

52. Jo YS, Oh HR, Kim MS, Yo NJ, Lee SH. Frameshift mutations of OGDH, PPAT and PCCA genes in gastric and colorectal cancers. Neoplasma. 2016;63(5): 681-6. https://doi.org/10.4149/neo_2016 504.

53. Chen YJ, Guo YN, Shi K, Huang HM, Huang SP, Xu WQ, et al. Downregulation of microRNA-144-3p and its clinical value in non-small cell lung cancer: a comprehensive analysis based on microarray, miRNA-sequencing, and quantitative real-time PCR data. Respir Res. 2019;20(1):48-65. https://doi. org/10.1186/s12931-019-0994-1.

54. Yao Y, Luo J, Sun $Q, X u T$, Sun $S$, Chen M, et al. HOXC13 promotes proliferation of lung adenocarcinoma via modulation of CCND1 and CCNE1. Am J Cancer Res. 2017;7(9):1820-34.

55. Liu H, Hu X, Zhu Y, Jiang G, Chen S. Up-regulation of SRPK1 in non-small cell lung cancer promotes the growth and migration of cancer cells. Tumor Biol. 2016;37(6):7287-93. https://doi.org/10.1007/s13277-015-4510-z.

56. Guide D. Stomach Cancer treatment choices by type and stage of stomach Cancer. NY: Am Cancer Soc; 2009.

57. Li T, Huang H, Shi G, Zhao L, Li T, Zhang Z, et al. TGF- $\beta 1-S O X 9$ axisinducible COL10A1 promotes invasion and metastasis in gastric cancer via epithelial-to-mesenchymal transition. Cell Death Dis. 2018;9(9):849-18. https://doi.org/10.1038/s41419-018-0877-2
58. Hu L, Duan YT, Li JF, Su LP, Yan M, Zhu ZG, et al. Biglycan enhances gastric cancer invasion by activating FAK signaling pathway. Oncotarget. 2014;5(7): 1885-96. https://doi.org/10.18632/oncotarget.1871.

59. Wang $P$, Wang $Y C$, Chen $X Y$, Shen $Z Y$, Cao H, Zhang YJ, et al. CTHRC1 is upregulated by promoter demethylation and transforming growth factor- $\beta 1$ and may be associated with metastasis in human gastric cancer. Cancer Sci. 2012;103(7):1327-33. https://doi.org/10.1111/j.1349-7006.2012.02292.x.

60. Yu J, Feng J, Zhi X, Tang J, Li Z, Xu Y, et al. Let-7b inhibits cell proliferation, migration, and invasion through targeting Cthrc1 in gastric cancer. Tumor Biol. 2015;36(5):3221-9. https://doi.org/10.1007/s13277-014-2950-5.

61. Zhang L, Xu Z, Xu X, Zhang B, Wu H, Wang M, et al. SALL4, a novel marker for human gastric carcinogenesis and metastasis. Oncogene. 2014;33(48): 5491-500. https://doi.org/10.1038/onc.2013.495.

62. Yuan $X$, Zhang $X$, Zhang W, Liang W, Zhang P, Shi H, et al. SALL4 promotes gastric cancer progression through activating CD44 expression. Oncogenesis. 2016;5(11):e268. https://doi.org/10.1038/oncsis.2016.69.

63. Kou YB, Zhang SY, Zhao BL, Ding R, Liu H, Li S. Knockdown of MMP11 inhibits proliferation and invasion of gastric cancer cells. Int J Immunopathol Pharmacol. 2013;26(2):361-70. https://doi.org/10.1177/ 039463201302600209

64. Collins CS, Hong J, Sapinoso L, Zhou Y, Liu Z, Micklash K, et al. A small interfering RNA screen for modulators of tumor cell motility identifies MAP4K4 as a promigratory kinase. Proc Nati Acad Sci. 2006;103(10):3775-80. https://doi.org/10.1073/pnas.0600040103.

65. Wang CY, Hua L, Sun J, Yao KH, Chen JT, Zhang JJ, et al. MiR-211 inhibits cell proliferation and invasion of gastric cancer by down-regulating SOX4. Int J Clin Exp Pathol. 2015;8(11):14013-20.

66. Lu Q, Ma X, Li Y, Song W, Zhang L, Shu Y, et al. Overexpression of FOXS1 in gastric cancer cell lines inhibits proliferation, metastasis, and epithelialmesenchymal transition of tumor through downregulating wnt/ $/$-catenin pathway. J Cell Biochem. 2019;120(3):2897-907. https://doi.org/10.1002/jcb.26821.

67. Shaha AR. TNM classification of thyroid carcinoma. World J Surg. 2007;31(5): 879-87. https://doi.org/10.1007/s00268-006-0864-0.

68. Hari DM, Leung AM, Lee JH, Sim MS, Vuong B, Chiu CG, et al. AJCC Cancer staging manual 7th edition criteria for colon cancer: do the complex modifications improve prognostic assessment? J Am Coll Surg. 2013;217(2): 181-90. https://doi.org/10.1016/j.jamcollsurg.2013.04.018.

69. Liu R, Zhong J, Yu X, Li Y, Chen P. Identifying critical state of complex diseases by single-sample-based hidden Markov model. Front Genet. 2019; 10:285-94. https://doi.org/10.3389/fgene.2019.00285.

70. Kullback S, Leibler RA. On information and sufficiency. Ann Math Stat. 1951; 22(1):79-86.

71. Shamilov A, Giriftinoglu C. Generalized entropy optimization distributions dependent on parameter in time series. WSEAS Trans on Inf Sci Appl. 2012; 1:102-11.

72. Oh JH, Gao J, Rosenblatt K. Biological data outlier detection based on Kullback-Leibler divergence. In 2008 IEEE International Conference on Bioinformatics and Biomedicine. IEEE; 2008. p. 249-54. https://doi.org/10. 1109/BIBM.2008.76.

73. Lindorff-Larsen $\mathrm{K}$, Ferkinghoff-Borg J. Similarity measures for protein ensembles. PLoS One. 2009;4(1):e4203. https://doi.org/10.1371/journal.pone.0004203.

74. Zhou SK, Chellappa R. From sample similarity to ensemble similarity: probabilistic distance measures in reproducing kernel hilbert space. IEEE Trans Pattern Anal Machine Intelligence. 2006;28(6):917-29. https://doi.org/ 10.1109/TPAMI.2006.120.

\section{Publisher's Note}

Springer Nature remains neutral with regard to jurisdictional claims in published maps and institutional affiliations. 